\title{
Characterization of microsatellite loci in ERysimum MEDIOHISPANICUM (BRASSICACEAE) AND CROSS-AMPLIFICATION IN RELATED SPECIES ${ }^{1}$
}

\author{
A. Jesús Muñoz-Pajares ${ }^{2,6}$, M. Belén Herrador ${ }^{3}$, Mohamed Abdelaziz ${ }^{2}$, \\ F. Xavier Picó ${ }^{4}$, Timothy F. Sharbel ${ }^{5}$, José M. Gómez ${ }^{3}$, And Francisco Perfectti ${ }^{2}$ \\ ${ }^{2}$ Departamento de Genética, Universidad de Granada, Granada, Spain; ${ }^{3}$ Departamento de Ecología, Universidad de Granada, \\ Granada, Spain; ${ }^{4}$ Departamento de Ecología Integrativa, Estación Biológica de Doñana (EBD), Consejo Superior de \\ Investigaciones Científicas (CSIC), Seville, Spain; and ${ }^{5}$ Apomixis Research Group, Leibniz-Institut für Pflanzengenetik und \\ Kulturpflanzenforschung (IPK), Gatersleben, Germany
}

- Premise of the study: We have developed and optimized microsatellite loci from a genomic library of Erysimum mediohispanicum. Microsatellites were also tested for cross-amplification in 31 other Erysimum species.

- Methods and Results: A total of 10 microsatellite loci were successfully amplified. They were polymorphic for 81 E. mediohispanicum individuals from two locations in Sierra Nevada (southeastern Spain), which showed similar patterns of genetic diversity. On average, microsatellites had 8.6 alleles per locus and an expected heterozygosity of 0.69 . Only one locus significantly departed from Hardy-Weinberg equilibrium in both locations. Most of the markers successfully amplified in other Erysimum species.

- Conclusions: The genetic attributes of microsatellite loci will allow their application to population genetic studies in Erysimum, such as genetic differentiation and structure, gene flow, pollinator-mediated speciation, and hybridization studies.

Key words: Brassicaceae; cross-amplification; Erysimum mediohispanicum; microsatellites.

Erysimum (Brassicaceae) is a genus comprising approximately 223 species (Al-Shehbaz et al., 2006) mainly distributed in the northern hemisphere. Erysimum mediohispanicum Polatschek is a biennial to perennial monocarpic herb endemic to the Iberian Peninsula, where it is distributed in two geographically distinct areas: one in northeastern and the other in southeastern Spain. Erysimum mediohispanicum typically exhibits high within- and among-population variation in floral traits, in particular corolla shape and size (Gómez et al., 2009a). It has been shown that different pollinators discriminate among different flower shapes, suggesting that phenotypic evolution and diversification can occur in this species (Gómez et al., 2008, 2009b). We have characterized 10 new polymorphic microsatellite loci for E. mediohispanicum and tested their cross-amplification in 31 other Erysimum species from different locations across Europe and Africa. These microsatellite loci will be primarily used for population genetics and gene flow studies in E. mediohispanicum populations characterized by different patterns of variation in floral shape and size as well as in the species' pollinator community.

\footnotetext{
${ }^{1}$ Manuscript received 18 April 2011; revision accepted 19 May 2011.

The authors thank the staff of Genetic Identification Services (Chatsworth, California, USA) for microsatellite development, and Leticia Ayllón-Egea and Yedra García-García for technical support. This study was funded by grants from Ministerio de Medio Ambiente y Medio Rural y Marino (MARM; 078/2007), Junta de Andalucía (P07-RNM-02869), Spanish Science Ministry (CGL 2009-07015), and Consolider-Ingenio (CSD2008-00040).

${ }^{6}$ Author for correspondence: ajesusmp@ugr.es
}

doi:10.3732/ajb.1100181

\section{METHODS AND RESULTS}

Microsatellite libraries were developed by Genetic Identification Services (Chatsworth, California, USA; http://www.genetic-id-services.com) following Jones et al. (2002). Genomic DNA was extracted using the DNeasy Plant Extraction kit (QIAGEN, Venlo, Netherlands) from one individual. Approximately $100 \mu \mathrm{g}$ of DNA were digested with RsaI, HaeIII, BsrB1, PvuII, StuI, ScaI, and EcoRV (New England Biolabs, Ipswich, Massachusetts, USA), and subsequent fragments were enriched in four motifs: CA-, AAC-, ATG-, and GA- using Biotin and Steptavidin magnetic beads for reversible capture (CPG, East Bank Demerara, Guyana). Resulting fragments were ligated into pUC19 plasmid and cloned into an E. coli strain DH5alpha (Invitrogen, Carlsbad, California, USA). After incubation, a total of 100 randomly chosen recombinant clones were selected, purified, and sequenced. Primer pairs were designed for 26 clones showing tandemly repeated motifs flanked by high quality sequence regions. Primer design was conducted using Designer PCR 1.03 (Research Genetics, Huntsville, Alabama, USA). Such primers were tested on a total of $81 E$. mediohispanicum individuals from two locations in southeastern Spain: El Dornajo $\left(37^{\circ} 7.67^{\prime} \mathrm{N}, 3^{\circ} 25.77^{\prime} \mathrm{W} ; N=30\right.$, UGR herbarium specimen: GDAC17407$1-2)$ and La Cortijuela $\left(37^{\circ} 4.66^{\prime} \mathrm{N}, 3^{\circ} 28.29^{\prime} \mathrm{W} ; N=51\right.$, UGR herbarium specimen: GDAC17421-1-2).

DNA was isolated from silica-dried leaf samples using the GenElute Plant Genomic DNA Miniprep kit (Sigma-Aldrich, St. Louis, Missouri, USA). PCR was performed in $15 \mu \mathrm{L}$ of reaction mixture containing $0.17 \mathrm{ng} / \mu \mathrm{L}$ of template genomic DNA, $1 \times$ buffer (ref. M0273S, New England BioLabs), $0.16 \mathrm{mM}$ each dNTP (Sigma-Aldrich), $0.33 \mu \mathrm{M}$ each forward (fluorescently tagged; Applied Biosystems, Foster City, California, USA) and reverse primer, and $0.02 \mathrm{U} / \mu \mathrm{L}$ Taq polymerase (ref. M0273S, New England Biolabs). PCR was conducted in a Gradient Master Cycler Pro S (Eppendorf, Hamburg, Germany) with an initial $30 \mathrm{~s}$ of denaturation at $94^{\circ} \mathrm{C}, 35$ cycles at $94^{\circ} \mathrm{C}$ for $15 \mathrm{~s}$, annealing temperatures $\left(T_{\mathrm{a}}\right.$; Table 1) for $30 \mathrm{~s}$, extension at $72^{\circ} \mathrm{C}$ for $30 \mathrm{~s}$, and a final extension at $72^{\circ} \mathrm{C}$ for 3 min. PCR products were diluted 1:15 and analyzed by MACROGEN analyzers (Geumchun-gu, Seoul, Korea; http://www.macrogen.com) using 400HD ROX (Applied Biosystems) as standard. Alleles were called using Peak Scanner Software version 1.0 (Applied Biosystems). 
TABLE 1. Characteristics of 10 microsatellite markers in Erysimum mediohispanicum. GenBank accession number, repeat motif, forward (F) and reverse (R) primers, F primer fluorescent tag, allele size ranges, and optimal annealing temperatures $\left(T_{\mathrm{a}}\right)$ are given.

\begin{tabular}{|c|c|c|c|c|c|}
\hline $\begin{array}{l}\text { Locus } \\
\text { (GenBank Accession No.) }\end{array}$ & Repeat motif & Primer sequence $\left(5^{\prime}-3^{\prime}\right)$ & Fluorescent label & Product size (bp) & $T_{\mathrm{a}}\left({ }^{\circ} \mathrm{C}\right)$ \\
\hline C5 (JF766210) & $\mathrm{CCA}_{8}$ & $\begin{array}{l}\mathrm{F}: \text { TCTTTCTTCTGCGGTTTATTC } \\
\mathrm{R}: \text { CGTTTTGTTGTGTTCTGG }\end{array}$ & 6-FAM & $164-182$ & 56 \\
\hline D2 (JF766211) & $\mathrm{CAT}_{23}$ & $\begin{array}{l}\mathrm{F}: \text { ACGGAAGATGACGATGATCGACTG } \\
\mathrm{R}: \text { CAATGTCCCTAATTGGTCAATGG }\end{array}$ & HEX & $117-189$ & 54 \\
\hline D4 (JF766212) & $\mathrm{ATC}_{7}$ & $\begin{array}{l}\text { F: TAAGGTGTTACCGGATTGTC } \\
\text { R: GTGACGATTCGCTCCTTG }\end{array}$ & NED & $200-215$ & 57 \\
\hline E4 (JF766213) & $\mathrm{CT}_{20}$ & $\begin{array}{l}\text { F: CCTTCCTCCGACTACTCTCC } \\
\text { R: TGAGCGACTGATGATGATTC }\end{array}$ & HEX & $145-178$ & 57 \\
\hline E8 (JF766214) & $\mathrm{CT}_{50}$ & $\begin{array}{l}\text { F: AGCTCACAGCCGTCGATGTTTGC } \\
\text { R: GAGGTGAAATACACGTAGAACCT }\end{array}$ & 6-FAM & $157-229$ & 50 \\
\hline D11 (JF766215) & $\mathrm{TCA}_{14}$ & $\begin{array}{l}F: \text { TCCAGGGTCTGAGTCAATATG } \\
\text { R: TTACCACTCCTTGCTTCTGAA }\end{array}$ & HEX & $179-197$ & 53 \\
\hline E6 (JF766216) & $\mathrm{TC}_{14}$ & $\begin{array}{l}\text { F: CTTGTAACCGAGCCACTCA } \\
\text { R: ATACGGAGAAGAAAGCGAATC }\end{array}$ & NED & $131-159$ & 53 \\
\hline D10 (JF766217) & $\mathrm{TCA}_{12}$ & $\begin{array}{l}\mathrm{F}: \text { ACTGCCATCAAACGACCTC } \\
\mathrm{R}: \text { TTGGTTGGAAAAGGGATTG }\end{array}$ & NED & $166-185$ & 53 \\
\hline E5 (JF766218) & $\mathrm{GA}_{13}$ & $\begin{array}{l}\mathrm{F}: \text { TCCATTTACACAATCCGTTCAT } \\
\mathrm{R}: \text { CCAACCTGACATCTTTGCTTC }\end{array}$ & 6-FAM & $167-195$ & 50 \\
\hline E3 (JF766219) & $\mathrm{GA}_{17}$ & $\begin{array}{l}\mathrm{F}: \text { TTCCTCCAGATGAAACTACACAGG } \\
\mathrm{R}: \text { ACTTACATCGGATCGGTTGAG }\end{array}$ & HEX & $215-253$ & 56 \\
\hline
\end{tabular}

Table 2. Genetic diversity of two populations of Erysimum mediohispanicum. Sample size $(N)$, number of observed alleles $\left(N_{\mathrm{a}}\right)$, allelic richness $\left(R_{\mathrm{s}}\right)$, observed heterozygosity $\left(H_{\mathrm{o}}\right)$, expected heterozygosity $\left(H_{\mathrm{e}}\right)$, and $P$ values for departure from Hardy-Weinberg equilibrium (HWE) are given for each microsatellite marker and population.

\begin{tabular}{|c|c|c|c|c|c|c|c|c|c|c|c|c|}
\hline \multirow[b]{2}{*}{ Locus } & \multicolumn{6}{|c|}{ El Dornajo } & \multicolumn{6}{|c|}{ La Cortijuela } \\
\hline & $N$ & $N_{\mathrm{a}}$ & $R_{\mathrm{s}}$ & $H_{\mathrm{o}}$ & $H_{\mathrm{e}}$ & HWE & $N$ & $N_{\mathrm{a}}$ & $R_{\mathrm{s}}$ & $H_{\mathrm{o}}$ & $H_{\mathrm{e}}$ & HWE \\
\hline C5 & 27 & 7 & 7.00 & 0.74 & 0.737 & 0.984 n.s. & 50 & 6 & 5.94 & 0.76 & 0.778 & 0.766 n.s. \\
\hline D2 & 29 & 19 & 18.50 & 0.72 & 0.925 & 0.057 n.s. & 50 & 17 & 16.87 & 0.78 & 0.891 & $0.041 *$ \\
\hline D4 & 30 & 7 & 6.60 & 0.50 & 0.629 & 0.277 n.s. & 51 & 6 & 5.92 & 0.59 & 0.640 & 0.567 n.s. \\
\hline E4 & 29 & 9 & 8.92 & 0.62 & 0.844 & 0.141 n.s. & 51 & 13 & 12.83 & 0.71 & 0.854 & $0.001 * *$ \\
\hline E8 & 29 & 10 & 9.93 & 0.31 & 0.861 & $0.000 * * *$ & 47 & 14 & 14.00 & 0.45 & 0.851 & $0.000 * * *$ \\
\hline D11 & 27 & 3 & 3.00 & 0.52 & 0.562 & 0.600 n.s. & 48 & 5 & 4.98 & 0.46 & 0.564 & $0.003 * *$ \\
\hline E6 & 29 & 7 & 6.93 & 0.72 & 0.664 & 0.920 n.s. & 50 & 8 & 7.87 & 0.68 & 0.696 & 0.993 n.s. \\
\hline D10 & 29 & 5 & 5.00 & 0.59 & 0.645 & 0.734 n.s. & 51 & 5 & 5.00 & 0.47 & 0.481 & 0.221 n.s. \\
\hline E5 & 28 & 12 & 11.89 & 0.71 & 0.870 & 0.798 n.s. & 51 & 12 & 11.68 & 0.75 & 0.797 & 0.131 n.s. \\
\hline E3 & 28 & 2 & 2.00 & 0.11 & 0.278 & $0.001 * *$ & 49 & 4 & 3.96 & 0.14 & 0.155 & 1.000 n.s. \\
\hline
\end{tabular}

Note: For HWE significance, n.s. $=$ not significant, $* P<0.05, * * P<0.01$, *** $P<0.001$.

Seven of the 26 tested primers failed to amplify, nine were monomorphic or showed complex patterns (primer sequences available upon request), and 10 were polymorphic (Table 1 ). We estimated the number of observed alleles per locus $\left(N_{\mathrm{a}}\right)$, allelic richness $\left(R_{\mathrm{s}}\right)$, observed heterozygosity $\left(H_{\mathrm{o}}\right)$, and expected heterozygosity $\left(H_{\mathrm{e}}\right)$ using FSTAT version 2.9.3 (Goudet, 1995). Linkage disequilibrium was tested with FSTAT using Bonferroni correction, and departures from Hardy-Weinberg equilibrium (HWE) were performed with GenAlEx version 6.3 (Peakall and Smouse, 2006).

For the El Dornajo population, the number of alleles per locus varied between two and 19, allelic richness between 2.0 and 18.5, observed heterozygosity between 0.11 and 0.74 , and expected heterozygosity between 0.28 and 0.93 (Table 2). For the La Cortijuela population, the number of alleles per locus varied between four and 17, allelic richness between 4.0 and 16.9, observed heterozygosity between 0.14 and 0.78 , and expected heterozygosity between 0.16 and 0.89 (Table 2). All loci showed no linkage disequilibrium $(P>0.004$ in all cases; nominal level $=0.0011)$. For El Dornajo and La Cortijuela, two and four out of 10 loci significantly departed from HWE, respectively (Table 2). However, only one out of 10 loci significantly departed from HWE in both locations (Table 2).

We analyzed the cross-amplification success of the 10 polymorphic microsatellite loci in a total of 31 Erysimum species (Appendix 1). Most of the loci amplified in several species. On average, each loci amplified in $79.1 \%$ (range $=$ 50.0-96.9\%) of the species (Appendix 1).

\section{CONCLUSIONS}

Genetic diversity parameters indicate that these microsatellite loci can be a useful tool to study neutral genetic variation in E. mediohispanicum populations. Questions like the relationship between genetic diversity and phenotypic diversity, the effects of geographical variation in pollinator abundance and diversity on genetic diversity, differentiation, and structure, or the extent of pollinator-mediated gene flow within and among populations can now be addressed. Given that most of the primers successfully amplified a band of the expected size in several Erysimum species, these microsatellites also have the potential to become an efficient molecular tool to address similar questions in other Erysimum species, as well as to explore speciation 
processes and contact zones commonly found in this highly diverse genus across its distribution range.

\section{LITERATURE CITED}

Al-Shehbaz, I. A., M. A. Beilstein, and E. A. Kellogg. 2006. Systematics and phylogeny of the Brassicaceae (Cruciferae): An overview. Plant Systematics and Evolution 259: 89-120.

Gómez, J. M., J. Bosch, F. Perfectti, J. D. Fernández, M. Abdelaziz, AND J. P. M. CAмACHO. 2008. Spatial variation in selection on corolla shape in a generalist plant is promoted by the preference patterns of its local pollinators. Proceedings of the Royal Society of London, B, Biological Sciences 275: 2241-2249.

Gómez, J. M., M. Abdelaziz, J. Muñoz-Pajares, and F. Perfectti. 2009a. Heritability and genetic correlation of corolla shape and size in Erysimum mediohispanicum. Evolution; International Journal of Organic Evolution 63: 1820-1831.

Gómez, J. M., M. Abdelaziz, J. P. M. Camacho, A. J. Muñoz-Pajares, AND F. PerfeCtTi. 2009b. Local adaptation and maladaptation to pollinators in a generalist geographic mosaic. Ecology Letters 12: 672-682.

GoudET, J. 1995. FSTAT (version 1.2): A computer program to calculate F-statistics. Journal of Heredity 86: 485-486.

Jones, K. C., K. F. Levine, And J. D. Banks. 2002. Characterization of 11 polymorphic tetranucleotide microsatellites for forensic applications in California elk (Cervus elaphus canadensis). Molecular Ecology Notes 2: 425-427.

Peakall, R., and P. E. Smouse. 2006. GenAlEx 6: Genetic analysis in Excel. Population genetic software for teaching and research. Molecular Ecology Notes 6: 288-295.

APPENDIX 1. Amplification of 10 highly polymorphic microsatellite markers in 31 Erysimum species (one individual per species).

\begin{tabular}{|c|c|c|c|c|c|c|c|c|c|c|}
\hline \multirow[b]{2}{*}{ Species } & \multicolumn{9}{|c|}{ Locus } & \multirow[b]{2}{*}{ E3 } \\
\hline & $\mathrm{C} 5$ & D2 & D4 & $\mathrm{E} 4$ & E8 & D11 & E6 & D10 & E5 & \\
\hline E. baeticum Polatschek subsp. baeticum & + & - & + & + & + & - & + & + & + & + \\
\hline E. bicolor DC. & + & - & + & + & + & + & + & + & + & + \\
\hline E. bonnanianum C. Presl & + & + & + & + & - & - & + & + & - & + \\
\hline E. collisparsum Jord. & + & + & + & + & - & + & + & + & - & + \\
\hline E. corinthium (Boiss.) Wettst. & + & + & + & + & + & + & + & + & + & - \\
\hline E. crepidifolium Rchb. & + & + & + & + & - & + & + & + & - & + \\
\hline E. duriaei Boiss. & + & + & + & + & + & - & + & + & + & + \\
\hline E. fitzii Polatschek & + & + & + & + & + & + & + & + & + & + \\
\hline E. gomezcampoi Polatschek & + & + & + & + & - & - & + & + & + & + \\
\hline E. gorbeanum Polatschek & + & + & + & + & - & + & + & + & + & + \\
\hline $\begin{array}{l}\text { E. incanum Kunze subsp. mairei (Sennen \& Mauricio) } \\
\text { Nieto Fel. }\end{array}$ & + & - & - & + & + & + & + & + & - & + \\
\hline E. jugicola Jord. & + & + & + & + & - & + & + & + & + & + \\
\hline E. merxmuellieri Polatschek & + & - & + & + & + & + & + & + & + & + \\
\hline E. metlesicsii Polatschek & + & + & + & + & - & + & + & - & - & - \\
\hline E. myriophyllum Lange & + & - & + & - & + & - & - & - & + & + \\
\hline E. myriophyllum var. cazorlense (Heyw.) Polatschek & + & + & + & + & + & + & + & + & + & + \\
\hline E. nervosum Pomel & + & - & + & + & + & - & - & - & + & - \\
\hline E. nevadense A. Heller & + & + & + & + & + & + & + & + & + & + \\
\hline E. odoratum Ehrh. & + & + & + & + & - & + & + & + & - & + \\
\hline E. penyalarense Polatschek & + & + & + & + & + & + & + & + & + & + \\
\hline E. popovii Rothm. & + & - & + & + & + & - & + & + & + & + \\
\hline E. pseudorhaeticum Polatschek & + & + & + & + & - & - & + & + & - & + \\
\hline E. rhaeticum DC. & + & + & + & + & - & + & + & + & - & + \\
\hline $\begin{array}{l}\text { E. riphaeanum Lorite, Abdelaziz, Muñoz-Pajares, } \\
\text { Perfectti \& J. M. Gómez }\end{array}$ & + & + & + & + & - & + & + & + & + & - \\
\hline E. rondae Polatschek & + & + & + & + & - & + & + & + & + & + \\
\hline E. ruscinonense Jord. & + & + & + & + & - & - & + & + & + & + \\
\hline E. scoparium Wettst. & + & + & + & + & - & + & + & + & + & + \\
\hline E. seipkae Polatschek & + & + & + & + & - & + & + & + & + & + \\
\hline E. semperflorens Wettst. & + & - & + & + & + & - & + & + & + & - \\
\hline E. sylvestre Scop. & + & - & + & + & - & - & + & - & - & + \\
\hline E. wilczekianum Braun-Blanq. \& Maire & - & - & - & - & + & - & + & - & - & - \\
\hline
\end{tabular}

Note: Plus and minus signs represent successful and unsuccessful amplifications, respectively. 